\title{
Effectiveness of novel combination chemotherapy, consisting of Temozolomide, Bevacizumab and Irinotecan as salvage chemotherapy regimen in the treatment of primary pediatric brain tumors
}

soheila zareifar ${ }^{1}$, Mehrpour Moradi $^{2}$, Mehran Karimi ${ }^{2}$, Amirhossein Najibi $^{3}$, and Mehrnaz Moradi ${ }^{3}$

${ }^{1}$ Shiraz University of medical sciences

${ }^{2}$ Shiraz University of Medical Sciences

${ }^{3}$ Fasa University of Medical Science

August 6, 2020

\begin{abstract}
Abstract Background: Primary pediatric brain tumors are the most prevalent type of childhood tumors and the most common cause of cancer death among children. However, there is insufficient literature regarding the use of novel chemotherapy agents to treat such tumors. Therefore, the purpose of this study is to evaluate the effectiveness of Irinotecan-based chemotherapy regimen for the treatment of primary brain tumors as well as minimizing morbidity and mortality rates. Procedure: In this cross-sectional study, 88 children aged 0 to 18 years old with primary brain tumors were investigated. Data was extracted from patients' medical records. 38 patients received Irinotecan treatment from the beginning (B) or after relapse (AB). At study termination, the response rate to the treatment as well as the Overall Survival (OS) and 6 months Progression Free Survival (6-mo-PFS) were estimated using Kaplan-Meier survival analysis and PFS estimation. Results: According to the findings of this study, pediatric brain tumors in males are more common than in females (60\% in comparison to $40 \%$ ). The highest incidence of brain tumors was reported in the age range of 6 to 12 years old. The findings of this study suggest that there is no statistically significant correlation between OS/6-mo-PFS and age, sex, tumor type, type of treatment and relapse. Conclusions: The administration of combination Irinotecan-based regimen leads to a high response rate and low toxicity in some types of primary pediatric brain tumors especially embryonal tumors, while it has little effect on others such as astrocytoma and ependymoma.
\end{abstract}

Effectiveness of novel combination chemotherapy, consisting of Temozolomide, Bevacizumab and Irinotecan as salvage chemotherapy regimen in the treatment of primary pediatric brain tumors

Soheila Zareifar M.D ${ }^{1}$, Mehrpour Moradi M.D ${ }^{1}$, Mehran Karimi M.D ${ }^{1}$, Amirhossein Najibi ${ }^{2}$, Mehrnaz Moradi $^{2}$

1. Hematology research center, Shiraz university of medical sciences, Shiraz, Iran

2. Student research committee, Fasa university of medical sciences, Fasa, Iran

*Correspondence to:

Mehrpour Moradi, MD, Hematology research center, Shiraz university of medical sciences, Shiraz, Iran, Tel.:0989173413740, Email: moradime@yahoo.com

Text word count: 3020 
Abstract word count: 246

Brief running title: Irinotecan therapy in pediatric brain tumors

Keywords: children, brain tumor, combination chemotherapy, Irinotecan

Tables: 6

Figures: 2

\begin{tabular}{ll}
\hline Abbreviations & Full phrase/term \\
\hline CNS & Central Nervous System \\
DPG & Diffuse Pontine Glioma \\
GBM & Glioblastoma Multiforme \\
HGG & High Grade Glioma \\
MRI & Magnetic Resonance Imaging \\
OR & Objective Response \\
OS & Overall Survival \\
PD & Progressive Disease \\
PFS & Progression Free Survival \\
SD & Stable Disease \\
WHO & World Health Organization \\
\hline
\end{tabular}

Abstract

Background:

Primary pediatric brain tumors are the most prevalent type of childhood tumors and the most common cause of cancer death among children. However, there is insufficient literature regarding the use of novel chemotherapy agents to treat such tumors.

Therefore, the purpose of this study is to evaluate the effectiveness of Irinotecan-based chemotherapy regimen for the treatment of primary brain tumors as well as minimizing morbidity and mortality rates.

\section{Procedure:}

In this cross-sectional study, 88 children aged 0 to 18 years old with primary brain tumors were investigated. Data was extracted from patients' medical records. 38 patients received Irinotecan treatment from the beginning (B) or after relapse (AB). At study termination, the response rate to the treatment as well as the Overall Survival (OS) and 6 months Progression Free Survival (6-mo-PFS) were estimated using KaplanMeier survival analysis and PFS estimation.

\section{Results:}

According to the findings of this study, pediatric brain tumors in males are more common than in females (60\% in comparison to 40\%). The highest incidence of brain tumors was reported in the age range of 6 to 12 years old. The findings of this study suggest that there is no statistically significant correlation between OS/6-mo-PFS and age, sex, tumor type, type of treatment and relapse.

\section{Conclusions:}

The administration of combination Irinotecan-based regimen leads to a high response rate and low toxicity in some types of primary pediatric brain tumors especially embryonal tumors, while it has little effect on others such as astrocytoma and ependymoma

\section{Introduction}


Despite remarkable advancements in the field of medicine, cancer remains one of the most important diseases ever. Second cause of death in most parts of the world, cancer is a disease that requires a fully dedicated medical care as well as social support. ${ }^{1,2}$

One out of every 600 children under the age of 16 struggles with cancer and it is estimated that $25 \%$ of these children have a central nervous system (CNS) tumor which is the most prevalent cause of mortality in children between the age of 3 and 7 years old. About $60 \%$ of patients who survive cancer face a life-threatening disability including cognitive and neuroendocrine system disabilities. ${ }^{3-7}$

Brain tumors, especially malignant ones, are among the most paralyzing, life threatening tumors because the patients not only suffer a definite incurability but also deal with a high risk of mortality. These tumors are categorized into 2 major types: primary and secondary. Primary tumors initially originate from the brain while secondary tumors are usually a metastasis of systemic malignancies. (2)

Pediatric Primary CNS tumors occur in 3.5-4.5 per 100000 persons with a sex ratio of 1.14 in which the male percentage varies in a range of 53.3 to 57.7 in different epidemiological studies. This is almost 0.1 of its incidences in adults. Conclusively, brain tumors are the most prevalent tumors and second most common malignancy (after Hematopoietic and lymphoid malignancies) in children with a generally higher incidence in males than females. ${ }^{7-10}$

Data from multiple national cancer registries depict differences in pediatric and adult brain tumor incidence. Overall, Medulloblastoma and low-grade glioma are the most common tumors in children under the age of 15 while in adults, high grade glioma and meningioma occur most often. ${ }^{3,11,12}$ The most prevalent Primary CNS tumor in children is Astrocytoma which includes low grade glioma, brainstem glioma and non-brainstem high grade glioma. Low grade glioma is the most common subdivision and responsible for $40 \%$ of pediatric brain tumors. Medulloblastoma and ependymoma are respectively in 2nd and 3rd place with regard to their incidence. ${ }^{8}$ Supratentorial and infratentorial tumors generally occur with an equal incidence; however, supratentorial tumors are more common under the age of 2 while infratentorial types have a higher incidence between the age of $4 \_10{ }^{5,13}$ Suprasellar and epiphyseal regions are typically potent areas for formation of supratentorial tumors. ${ }^{8}$ In comparison with adults, pediatric infratentorial tumors mainly form in the hind brain (Rhombencephalon) and lower regions such as brainstem. Depending on their type, size and anatomical location, as well as the age of onset, these tumors may have a variety of different initial symptoms.

Based on tumor's anatomical location, the most common initial symptoms are increased intracranial pressure in supratentorial tumors, nausea and vomiting in hind brain tumors $(75 \%)$, headache in tumors of cerebral hemispheres $(49 \%)$ and backache in spinal tumors $(67 \%){ }^{4}$

Considering the increased occurrence of brain tumors in all age groups and its significant mortality rate in children, an implementation of highly accurate medical modalities such as surgery, radiotherapy and chemotherapy is necessary for early diagnosis and treatment of the disease. Since currently there is no complete cure for brain tumors, it is of great importance to try to increase patients' survival rates and ameliorate their painful symptoms. ${ }^{2,5,14}$ There are a variety of treatment strategies based on the type, location, removability, growth, relapse chances and clinical manifestations of the tumor. In low grade glioma, which is completely removable by surgery, the average 10-year survival rate after surgery is $90 \_100 \%$. At the same time, despite surgery and consequent radiotherapy in recurring or irremovable high-grade tumors, treatment with available chemotherapy regimens has little effect on increasing the Overall Survival rate. In case of relapse, chemotherapy can only exert a restricted effect on patients' overall survival improvement. ${ }^{5,14-16}$

With the challenges mentioned above, there is an urge for focusing on new pharmaceutical agents and novel therapeutic approaches to cancer treatment among which is the life-saving chemotherapy regimen based on a camptothecin derivative agent named Irinotecan which acts as a topoisomerase- 1 inhibitor. Irinotecan can be used mono-therapically or in combination with other synergic agents such as Temozolomide or Bevacizumab to overcome the resistance of tumor cells especially in adults. ${ }^{14,17}$

Considering the fact that there isn't, yet, an adequate number of comprehensive studies about the synergistic 
effects of this new agent and there are only a restricted number of clinical trials on this subject, the aim of this study is to investigate the efficacy and efficiency of Irinotecan based chemotherapy regimen in combination with Bevacizumab in a target group of children.

\section{Patients and methods}

In this cross-sectional study, 88 children with primary brain tumor who referred to Amir Oncology Hospital during the years 2008_2018 were studied based on the available clinical protocols. All data including age, sex, tumor type and size, initial symptoms, location of the tumor, MRI history preceding and following surgery, type of surgery, type of the chemotherapy regimen and the objective response rate (ORR)were extracted from the patients' medical records. Patients who hadn't received the Irinotecan based regimen since the onset of the tumor or after the relapse, were excluded from this study.

All patients underwent brain and spinal cord MRI in the first Chemotherapy session and this was repeated every 3_6 months during the treatment course. The average tumor size was measured through the Bidirectional(2D) Area method and the correlated response rates were determined based on the McDonald Criteria that was suggested for the quantitative evaluation of ORR in 1990.

According to the McDonald Criteria, a total tumor signal termination in brain MRI indicates a Complete Response(CR) while a reduced tumor size by more than $50 \%$ indicates a Partial Response(PR) and a decrease or increase in tumor size by $0 \_25 \%$ is referred to as a Stable Disease (SD) while an increase by more than $25 \%$ shows a Progressive Disease(PD).

In this study, patients received different chemotherapy regimens based on their tumor size, tumor type and its functionality, anatomical location of the tumor, tumor's grade determined by the WHO system and other related parameters.

The regimens are as follows:

1)Regimen A which consists of Vincristine, Lomustine (CCNU) and Cisplatin.

2)Regimen B which consists of Irinotecan, Temozolomide,Bevacizumab and Vincristine

Moreover, patients who received none of the above are shown with $\mathrm{W}$ and those who received both regimens sequentially are labeled as AB. Also, patients who received a regimen else than A at first and then received regimen $\mathrm{B}$ are labeled as $\mathrm{WB}$.

The collected data were analyzed using SPSS V.19 and Pearson's correlation test, Kaplan-Meier analysis and descriptive statistics including Central Tendency, Frequency Distribution and Distribution Ratio, as it is noticed in the respective tables and charts. A P_value of less than 0.05 was considered statistically significant.

\section{Results}

In this section, we will take a detailed look at the descriptive statistics of patients' demographic characteristics. We have used measures of central Tendency, frequency distribution, distribution rate and measures of variability to describe the data.

\section{Measures of pediatric brain tumor}

Out of 88 patients with brain tumor, 53 patients were male which equals 60 percent of the target population. Other quantitative data classifying tumors' distribution rate based on their type, are reflected in Fig. 1.

\section{Frequency based on age group}

Patients were divided into 4 age groups including: Infants (0_2 years old), young children (2_6 years old), children (6_12 years old) and adolescents (12_18 years old). Frequency and percentage of these groups are respectively demonstrated in Fig. 2. As seen in the figure, children have the highest and adolescents have 
the lowest frequency. Additionally, the average age of the patients were 6.5 years with a minimum age of 4 months and a maximum age of 16 years.

\section{Tumor type distribution sorted by age group}

The study of tumor frequency based on age groups showed a different distribution pattern in each group; in a way that the most common tumor type was ependymoma among infants, Astrocytoma among young children and embryonic tumor among children and adolescents. (see table 1)

\section{Frequency based on anatomic location}

The data in this study depicted that the highest percentage of the investigated tumors occurs in infratentorial region of the brain (including cerebellum, brainstem, hind brain and ventricles) with a percentage of $82 \%$ while the lowest percentage was in spinal regions with a percentage of $4 \%$. Moreover, in supratentorial regions (including cerebral lobes, thalamus, hypothalamus and suprasellar masses) the percentage was $14 \%$.

\section{Frequency distribution of the initial clinical manifestations}

Investigating the early symptoms of the tumors as initial manifestations, headache was the most frequent symptom. Vomiting, ataxia and double vision were respectively indicated as the next most frequent symptoms after headache, as shown in more details in table 2. Table 3 demonstrates the frequency of initial tumor manifestations classified by tumor's anatomical location. Therefore, headache, vomiting, ataxia and double vision have the highest frequency in infratentorial region while in supratentorial region, seizure, horizontal nystagmus and headache are respectively the most frequent symptoms.

\section{Frequency distribution of tumor-induced morbidities}

Out of all 88 patients, 57 patients $(65 \%)$ did not report any morbidity while 31 did experience different types of morbidity in different levels. The most prevalent morbidities are respectively pointed out to as seizure $(40 \%)$, ataxia (30\%), walking disability $(20 \%)$ and strabismus $(10 \%)$.

\section{Frequency distribution of chemotherapy regimens}

The frequencies of the chemotherapy regimens applied in this study are shown in table 4 . The regimens include regimen A (composed of Vincristine, CCNU and Cisplatin) and regimen B (composed of Irinotecan, Temozolomide, Bevacizumab and Vincristine). Moreover, patients who received none of the above are shown with $\mathrm{W}$ and those who received both regimens sequentially are labeled as AB. Also, patients who received a regimen else than $\mathrm{A}$ at first and then received regimen $\mathrm{B}$ are labeled as WB. Regimen $\mathrm{A}$ has the highest regimen frequency while WB has the lowest one.

According to McDonald criterion ${ }^{11}$ (see supporting information, supplemental table 1), we categorized frequency and response rate of regimens in studied tumor into 5 groups based on MRI reports (table 5). With due attention to table 5 , the most obvious response observed was complete response $(\mathrm{CR})$ and minor response(MR) had the lowest prevalence amongst patients.

Progression free survival (PFS) was $55 \%$ in patients who have received salvage regimen after standard regimen $(\mathrm{AB})$ and $38.5 \%$ in patients who received salvage regimen from the beginning(B). Although PFS is quite different in these two groups, this difference is not statistically significant (See table 6).

We also studied 6-month PFS in patients receiving salvage regimen based on their tumor type. Patients who had embryonal tumors and astrocytic tumors respectively had the longest and the shortest survival (see supporting information, supplemental table 2).

The PFS is $60 \%$ in male and $41.7 \%$ in female patients. Although there is a remarkably higher PFS in males than females, there is no meaningful relationship between 6-mon-PFS and sex (see supporting information, supplemental table 3).

Patients who received the salvage regimen after treatment with standard regimen $(\mathrm{AB})$ depicted a higher survival rate in comparison with the other two groups. However, after survival analysis through the Kaplan- 
Meier procedure, there was no statistically significant correlation between survival and type of salvage regimen (Log Rank P-value=0.087). In addition, other factors previously proved to affect survival in children with primary brain tumor, such as age, sex and tumor type were analyzed with the Kaplan-Meier procedure to inspect their effect on patients' survival during the course of treatment (see supporting information, supplemental table 4).

There was no significant relationship between age and survival despite adolescent and infant 2-year survivals being higher than other age groups. Although survival rate for females was higher than males (1.838 and 1.700), this difference was not of statistical significance. Patients with ependymomal tumors had the longest estimated survival time (2 years) and the highest survival rate (90\%). Nevertheless, tumor type was not meaningfully correlated with survival (see supporting information, supplemental tables 5,6 and 7).

\section{Conclusion and discussion}

Once again, as we already mentioned in previous sections, the incidence of brain tumors is higher in males (60\%) than in females (40\%). This fact can also be observed in different subdivisions of brain tumors as in that embryonal and ependymomal tumors are more prevalent in males than females. However, astrocytic tumors have an almost similar occurrence in both sexes. The dominance of brain tumor incidence in males compared to females is a recognized fact in which factors such as the role of sex hormones and sex chromosomes are highly important. ${ }^{18}$

The analysis of the collected data in this study showed that the incidence of pediatric brain tumors is highest in child (6_12) and young child (2_6) age group, with a peak in the average age of 6.5 years old. This finding is compatible with the findings of Golshani et. all study conducted in 1997_2015. ${ }^{10}$ The most prevalent primary brain tumors were embryonal (especially medulloblastoma) and astrocytoma (especially low-grade glioma), acknowledging the findings of Wells' et all study. ${ }^{8}$

Infratentorial tumors are more prevalent in comparison with supratentorial and spinal tumors especially in children below the age of 12 . This is compatible with the findings of Gottardo et all in 2008. ${ }^{5}$

However, this study also showed that supratentorial tumors are more common in the infant age group, which is in contrast with Gottardo's findings. ${ }^{5}$ The most prevalent initial manifestations of brain tumors, with respect to their frequency percentage, were : headache(31.7\%),vomiting $(26.2 \%)$,ataxia(12.7\%) and double vision(5.6\%). In a similar study conducted by Zali et all, vomiting, headache and ataxia were respectively the most prevalent initial symptoms. In another metanalysis carried out by Wilen et all, headache, vomiting and ataxia were sequentially the most prevalent symptoms. This high incidence of the three mentioned symptoms could be related to the increased intracranial pressure with a precise focus on the cerebellum, a part of the brain that has a key role in regulating balance and voluntary movement. ${ }^{4}$

In the current study, out of 88 patients with pediatric brain tumors, 38 persons received the Irinotecan based lifesaving regimen, 8 of which had embryonal tumors, 20 had Astrocytic and 10 had ependymomal tumors. The remaining 50 patients received either other standard regimens or a non-Irinotecan containing regimen. The medical responses of those 38 patients were recorded and investigated.

Out of the 38 patients mentioned above, 23 received the lifesaving regimen due to a lack of response to other standard regimens (20 received $\mathrm{AB}$ and 3 received $\mathrm{WB}$ regimen) and the other 15 patients received the lifesaving regimen( regimen B) from the beginning due to the inoperability of the tumor or its histological nature and its high grade of invasiveness. Half of those who received the AB regimen had an ependymomal tumor while the other half suffered Astrocytic or embryonal tumors. Of the 15 patients who received regimen B, 13 patients suffered Astrocytic tumors, a majority of which were DPG and HGG tumors.

Ultimately, among the 20 patients who received AB regimen, PFS was $55 \%$ with a SE of 11.1 while in the 15 patients who received regimen B from the beginning PFS at the end of 6 months was $38.5 \%$ with a SE of 13.5. The reason behind a lower OS and PFS in patients who received B regimen compared to those who received $\mathrm{AB}$, is the high-grade nature of Glioma tumors and their highly risky location near the brainstem. 
In this case, Vredenburg et all came up with similar conclusions in a study conducted between January and December 2007. ${ }^{14,19}$

In a study conducted by Hummel Trent et all between the years 2000 and 2013, 27 patients of whom, 15 had DIPG and 12 had HGG, with an average age of 10-year-old were studied. Patients received Bevacizumab, Irinotecan and Temozolomide either due to an inadequate response to other standard chemotherapy regimens or due to relapse. Conclusively, three-year PFS and OS were respectively $33 \%$ and 50\%, which are similar results to the findings of the current study. ${ }^{20}$

In another study conducted by Zhang et all during the years 2000_2011, 480 patients with Glioblastoma multiforme (GBM) were studied. 183 patients received only a Bevacizumab containing regimen while the others (397 patients) received a combination of Bevacizumab and Irinotecan. The average OS for the first group (only Bevacizumab) was 8.63 months and 8.91 for the second group (Bevacizumab and Irinotecan combination). Although the results of Zhang's study were similar to the current study, adding Irinotecan to the Bevacizumab regimen didn't make a statistically significant difference in the 6_month PFS and OS. ${ }^{21}$

The findings of this study suggest that embryonal tumors have an average PFS and OS of $62.5 \%$ while ependymomal tumors have a PFS of $60 \%$ and an OS of $90 \%$. Furthermore, Astrocytic tumors have a PFS of $47.4 \%$ and an OS of $65 \%$, which is compatible with the suggested PFS for high grade Astrocytic tumors $(38 \%)$ in Parekh et all study. ${ }^{22}$

In this cross-sectional study, 8 patients with relapsing medulloblastoma received the $\mathrm{AB}$ regimen and 2 patients received B regimen from the beginning, all of whom had an average PFS and OS of $62.5 \%$ which is the highest OS among all three major tumor subdivisions. This is compatible with the findings of Aguilera et all study and suggests that the combination of Bevacizumab and Irinotecan, with or without Temozolomide, provides the most effective response with the lowest toxicity in children with relapsing medulloblastoma.$^{23}$

However, when Grill et all conducted a research in 2013 in which they studied 66 patients with treatment resistant medulloblastoma and applied Temozolomide and Irinotecan regimens, an average PFS of only $37 \%$ was recorded. This does not match with the findings of this study. ${ }^{24}$

Patients who received the $\mathrm{AB}$ regimen following an application of standard regimens had a higher PFS and OS (55\% and $85 \%)$, compared with those who received the B regimen from the very beginning (38.5\% and $50 \%$ ). Nevertheless, this difference was not statistically significant, matching the findings of Vredenburgh et all study. ${ }^{14}$

Research limitations: 1. Deficiencies in medical records to which a better dedication is required 2 . Patients' not referring to the hospital for further evaluations, so that contacting them through phone calls was a necessity 3. Difficult access to radiology series and surgical records 4. A necessity for further research and investigations, considering the growing incidence of primary pediatric brain tumors and lack of information in its respective field

\section{Conflict of Interest Statement}

The authors hereby confirm that they have no conflicts of interest in any shape or form that can possibly arise controversy.

\section{Acknowledgments}

The authors would like to acknowledge all those who participated in/contributed to this study including patients, medical care professionals, laboratory staff and any other individual who has played a role, partially or substantially, in conducting this study.

\section{Data Availability Statement}

The data that support the findings of this study are available from the corresponding author upon reasonable request. 


\section{References}

1. Mousavi sm, Pourfeizi A, Dastgiri S. Childhood Cancer in Iran.Journal of pediatric hematology/oncology. 2010;32:376-382.

2. pakpour haji agha a, yekani nejhad ms, khoshnevisan a, nikobakht m, mardani a, kamali ardakani s. Health related quality of life associated factors among patients with brain tumors. journal of ilam university of medical sciences. 2013;21(3):1-11.

3. Wilne S, Koller K, Collier J, Kennedy C, Grundy R, Walker D. The diagnosis of brain tumours in children: a guideline to assist healthcare professionals in the assessment of children who may have a brain tumour. Arch Dis Child. 2010;95(7):534-539.

4. Wilne S, Collier J, Kennedy C, Koller K, Grundy R, Walker D. Presentation of childhood CNS tumours: a systematic review and meta-analysis. Lancet Oncol. 2007;8(8):685-695.

5. Gottardo NG, Gajjar A. Chemotherapy for malignant brain tumors of childhood. $J$ Child Neurol. 2008;23(10):1149-1159.

6. Guerreiro Stucklin AS, Ramaswamy V, Daniels C, Taylor MD. Review of molecular classification and treatment implications of pediatric brain tumors. Curr Opin Pediatr. 2018;30(1):3-9.

7. Mehrazin M, Yavari P. Morphological pattern and frequency of intracranial tumors in children. Child's Nervous System.2007;23(2):157-162.

8. Wells EM, Packer RJ. Pediatric brain tumors. Continuum (Minneap Minn). 2015;21(2 Neurooncology):373-396.

9. Bondy ML, Scheurer ME, Malmer B, et al. Brain tumor epidemiology: consensus from the Brain Tumor Epidemiology Consortium. Cancer.2008;113(7 Suppl):1953-1968.

10. Aghayan Golkashani H, Hatami H, Farzan A, et al. Tumors of the Central Nervous System: An 18-Year Retrospective Review in a Tertiary Pediatric Referral Center. Iran J Child Neurol. 2015;9(3):24-33.

11. Wen PY, Macdonald DR, Reardon DA, et al. Updated response assessment criteria for high-grade gliomas: response assessment in neuro-oncology working group. J Clin Oncol. 2010;28(11):1963-1972.

12. Pollack IF. Pediatric brain tumors. Seminars in Surgical Oncology. 1999;16(2):73-90.

13. Echevarría ME, Fangusaro J, Goldman S. Pediatric central nervous system germ cell tumors: a review. The oncologist.2008;13(6):690-699.

14. Vredenburgh JJ, Desjardins A, Reardon DA, Friedman HS. Experience with irinotecan for the treatment of malignant glioma.Neuro-oncology. 2009;11(1):80-91.

15. Bouffet E, Capra M, Bartels U. Salvage chemotherapy for metastatic and recurrent ependymoma of childhood. Child's Nervous System.2009;25(10):1293.

16. Friedman GK, Spiller SE, Harrison DK, Fiveash JB, Reddy AT. Treatment of children with glioblastoma with conformal radiation, temozolomide, and bevacizumab as adjuncts to surgical resection. Journal of pediatric hematology/oncology. 2013;35(3):e123-e126.

17. Wagner L, Turpin B, Nagarajan R, Weiss B, Cripe T, Geller J. Pilot study of vincristine, oral irinotecan, and temozolomide (VOIT regimen) combined with bevacizumab in pediatric patients with recurrent solid tumors or brain tumors. Pediatric blood $\&$ cancer.2013;60(9):1447-1451.

18. Sun T, Warrington NM, Rubin JB. Why does Jack, and not Jill, break his crown? Sex disparity in brain tumors. Biology of sex differences. 2012;3(1):3. 
19. Zaky W, Wellner M, Brown RJ, Blüml S, Finlay JL, Dhall G. Treatment of children with diffuse intrinsic pontine gliomas with chemoradiotherapy followed by a combination of temozolomide, irinotecan, and bevacizumab. Pediatric hematology and oncology.2013;30(7):623-632.

20. Hummel TR, Salloum R, Drissi R, et al. A pilot study of bevacizumab-based therapy in patients with newly diagnosed high-grade gliomas and diffuse intrinsic pontine gliomas. Journal of neuro-oncology. 2016;127(1):53-61.

21. Zhang G, Huang S, Wang Z. A meta-analysis of bevacizumab alone and in combination with irinotecan in the treatment of patients with recurrent glioblastoma multiforme. Journal of clinical neuroscience. 2012;19(12):1636-1640.

22. Parekh C, Jubran R, Erdreich-Epstein A, et al. Treatment of children with recurrent high grade gliomas with a bevacizumab containing regimen. Journal of neuro-oncology. 2011;103(3):673-680.

23. Aguilera D, Mazewski C, Fangusaro J, et al. Response to bevacizumab, irinotecan, and temozolomide in children with relapsed medulloblastoma: a multi-institutional experience. Child's Nervous System.2013;29(4):589-596.

24. Grill J, Geoerger B, Gesner L, et al. Phase II study of irinotecan in combination with temozolomide (TEMIRI) in children with recurrent or refractory medulloblastoma: a joint ITCC and SIOPE brain tumor study.Neuro-oncology. 2013;15(9):1236-1243.

\section{Figures Legends}

Figure 1 Frequency percentage of different types of pediatric brain tumors

Figure 2 frequency distribution percentage of the studied tumors based on age group

\section{Hosted file}

Table 1.docx available at https://authorea.com/users/349148/articles/474252-effectivenessof-novel-combination-chemotherapy-consisting-of-temozolomide-bevacizumab-and-irinotecanas-salvage-chemotherapy-regimen-in-the-treatment-of-primary-pediatric-brain-tumors

\section{Hosted file}

Table 2.docx available at https://authorea.com/users/349148/articles/474252-effectivenessof-novel-combination-chemotherapy-consisting-of-temozolomide-bevacizumab-and-irinotecanas-salvage-chemotherapy-regimen-in-the-treatment-of-primary-pediatric-brain-tumors

\section{Hosted file}

Table 3.docx available at https://authorea.com/users/349148/articles/474252-effectivenessof-novel-combination-chemotherapy-consisting-of-temozolomide-bevacizumab-and-irinotecanas-salvage-chemotherapy-regimen-in-the-treatment-of-primary-pediatric-brain-tumors

\section{Hosted file}

table 4.docx available at https://authorea.com/users/349148/articles/474252-effectivenessof-novel-combination-chemotherapy-consisting-of-temozolomide-bevacizumab-and-irinotecanas-salvage-chemotherapy-regimen-in-the-treatment-of-primary-pediatric-brain-tumors

\section{Hosted file}

Table 5.docx available at https://authorea.com/users/349148/articles/474252-effectivenessof-novel-combination-chemotherapy-consisting-of-temozolomide-bevacizumab-and-irinotecanas-salvage-chemotherapy-regimen-in-the-treatment-of-primary-pediatric-brain-tumors

\section{Hosted file}


Table 6.docx available at https://authorea.com/users/349148/articles/474252-effectivenessof-novel-combination-chemotherapy-consisting-of-temozolomide-bevacizumab-and-irinotecanas-salvage-chemotherapy-regimen-in-the-treatment-of-primary-pediatric-brain-tumors

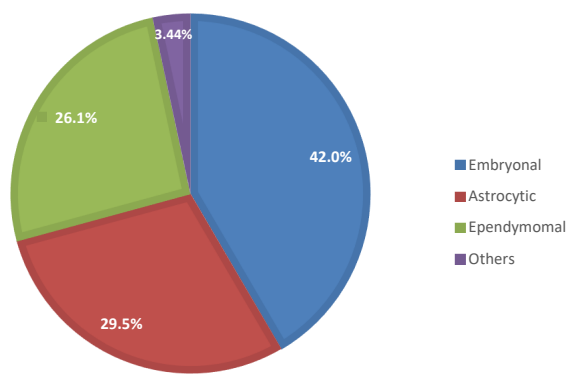




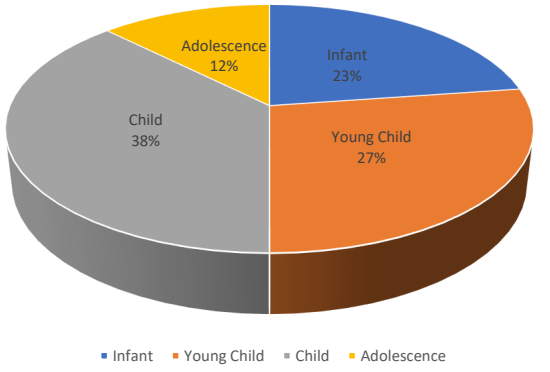

